Ahluwalia, A.

\section{Methylation: a predictor of chemotherapy response in ovarian cancer?}

\author{
A. Ahluwalia', P. Yan², T.H.-M. Huang ${ }^{2}$ \& K.P. Nephew ${ }^{1}$ \\ ${ }^{1}$ Medical Sciences, Indiana University School of Medicine, Bloomington, \\ Indiana 47405, USA \\ ${ }^{2}$ Department of Pathology, Ellis Fischel Cancer Center, University of \\ Missouri-Columbia, Missouri 65203, USA
}

Little is known about the molecular determinants of ovarian cancer, the fifth most common cause of cancer death in women. CpG island (CGI) hypermethylation is known to turn off gene expression and is associated with cancer progression. Our group used differential methylation hybridization, a microarraybased technique, to scan for alterations in methylation in ovarian cancer. We performed such screening of stage IIIC epithelial ovarian carcinomas $(N=10)$, normal ovarian surface epithelial cells and ovarian cancer cell lines. A wide range of hypermethylated CGIs $(2.5$-fold increase; $P<0.05)$ was seen in the ovarian carcinomas (23-98); however, the number of hypermethylated CGIs was similar across ovarian cancer cell lines (38-51). There were 12 hypermethylated CGIs $(P<0.05)$ common to the ovarian cancer cell lines and tumors. Sequencing analysis revealed 11 methylated CGI tags in the ovarian tumors that had previously been shown to be hypermethylated in breast cancer. Tumor methylation levels before therapy were associated with patient response to chemotherapy $(P=0.048$; one-sided exact Wilcoxon rank sum test). Patients displaying a complete response had tumors with a low methylation score; conversely, patients with progressive disease had tumors with a higher methylation score. Although we have analyzed only a small fraction $(2 \%)$ of the $\mathrm{CpG}$ islands in the human genome, we have found a relationship between the amount of methylation in ovarian tumors and response to chemotherapy. Our proof-of-concept study lays the foundation for genome-wide screening of methylation using DMH to examine epigenotypephenotype relationships in ovarian cancer.

Ali, Shujath

\section{Genomic approaches to the development of prostate cancer diagnostics}

Shujath Ali, Yongming Sun, Carrie Drumright, Luis Carretero, Christina Tran, Anton Nguyen, Dan Medynski, Yan Liu, Vu Viet Hoang, Brandon Liang, Herve Recipon, Robert Cafferkey \& Roberto Macina

diaDexus, Inc., 3303 Octavius Drive, Santa Clara, California 95054, USA

Using bioinformatic and genomic approaches, we have identified several candidate genes that are differentially expressed in prostate cancer. The LifeSeq (Incyte Pharmaceuticals) DNA expressed sequence tag database consists of more than 6.4 million tags obtained from 1,317 complementary DNA libraries made from various tissues (normal and diseased). Using a bioinformatic approach, we looked for candidate genes that show a strong specificity for expression in prostate tissue and also a significant upregulation in cancerous tissue compared with normal tissue. In parallel we constructed several subtraction cDNA libraries using suppressive hybridization protocols (Clontech). These libraries are being sequenced and analyzed. To date a total of 81 genes have been chosen as candidates for further expression analysis. Expression analysis of these candidate genes involved the use of a sensitive Taqman quantitative polymerase chain reaction assay that further determined the tissue-specific expression and whether higher levels of expression are indicative of prostate cancer. Expression analysis data using RNA from different tissues and disease states (approximately 200 samples) helped rank these candi- dates. A total of eight candidate genes (Arg2, Pro101, Pro108, Pro111, Pro118, Pro119, Pro121, Pro130) were selected for further analysis. A majority of these have been cloned and expressed in bacteria. Monoclonal antibodies are available for two of them, and immunoassays will be developed shortly. These candidates, either individually or grouped in panels, have the potential to become new prostate cancer biomarkers for early detection, differential diagnosis, disease monitoring and disease surveillance.

Allander, Susanne

[6]

\section{Microarray-based genetic analysis of synovial sarcomas}

\author{
Susanne Allander ${ }^{1}$, Yidong Chen ${ }^{1}$, Marc Ladanyi ${ }^{2}$ \\ $\&$ Paul Meltzer ${ }^{1}$ \\ ${ }^{1}$ Cancer Genetics Branch, National Human Genome Research Institute, \\ National Institutes of Health, Bethesda, Maryland, USA \\ ${ }^{2}$ Department of Pathology, Memorial Sloan-Kettering Cancer Center, New York, \\ New York, USA
}

Soft-tissue sarcomas are tumors of mesenchymal origin that often constitute a diagnostic and therapeutic dilemma. Some soft-tissue sarcomas are characterized by specific chromosomal translocations. The resulting fusion transcripts typically encode aberrant transcription factors, and their pathogenic effects are likely to be mediated through their actions on gene expression. Based on this hypothesis we are investigating gene expression patterns in synovial sarcoma using complementary DNA microarrays containing 6,500 sequence-verified human cDNAs. The $\mathrm{t}(\mathrm{X} ; 18)(\mathrm{p} 11.2 ; \mathrm{q} 11.2)$ translocation is characteristic of synovial sarcomas and fuses the SYT gene to either SSX1 or SSX2. Expression analyses were performed on 16 synovial sarcomas confirmed to have either the SYT-SSX1 or SYT-SSX2 fusion transcript. Five other sarcoma samples, mostly malignant fibrous histiocytomas, were included as a comparison group. Hierarchical clustering analysis shows that these two tumor groups clearly separate with distinct expression patterns. One of the tumors, previously diagnosed as a synovial sarcoma, was closer to the malignant fibrous histiocytoma cluster. Analysis by means of the polymerase chain reaction with reverse transcription showed that this tumor was lacking the SYT-SSX fusion transcript, and the histological review reclassified it as a fibrosarcoma. This indicates that SYT-SSX-verified synovial sarcomas indeed have a specific expression profile. We are now attempting to determine the genes best able to define the synovial sarcoma cluster. Synovial sarcomas have two major histological subtypes, biphasic and monophasic, defined by the presence or absence of glandular epithelial differentiation in a background of spindle cells. Analyses of the genes discriminating monophasic from biphasic synovial sarcomas may also provide clues to the pathways regulating epithelial differentiation.

Alsner, Jan

\section{Detection of TP53 mutations by denaturing high-performance liquid chromatography}

\author{
Jan Alsner, Susanne Berg Sorensen, Jesper Grau Eriksen \\ $\&$ Jens Overgaard \\ Department of Experimental Clinical Oncology, Aarhus University Hospital, \\ Aarhus, Denmark
}

Recent insight into the clinical behavior of tumors harboring tumor protein p53 (TP53) mutations indicates that information concerning TP53 can be useful in diagnosis, prognosis and choice of therapy for certain cancers. To detect mutations in clinical material, highly specific and sensitive mutation detection methods are 
needed; these are often laborious and not practical for high-throughput screening. In order to develop a rapid automated mutation detection method, we have evaluated denaturing high-performance liquid chromatography for detection of TP53 mutations in tumor tissue. The specificity and sensitivity of the method were compared to those of denaturing gradient gel electrophoresis through testing on standard-quality DNA preparations as well as on DNA extracted from formalin-fixed, paraffin-embedded tissue sections.

Amler, Lukas

\section{Functional dissection of transcriptional profiles in androgen-dependent and - independent prostate cancer}

Lukas Amler ${ }^{1}$, David Agus ${ }^{2}$, Nicholas Dracopoli ${ }^{1}$, Howard Scher ${ }^{2} \&$ Garret Hampton ${ }^{3}$

${ }^{1}$ Bristol-Myers Squibb, Princeton, New Jersey, USA

${ }^{2}$ Memorial Sloan-Kettering Cancer Center, New York, New York, USA

${ }^{3}$ Genomics Institute of the Novartis Research Foundation, La Jolla,

California, USA

Using microarray analysis, we have measured changes in gene expression that occurred during the evolution of a primary prostate cancer xenograft, CWR22, from normalcy to relapsed, androgen-independent growth following androgen deprivation therapy. Rather than relying on simple pairwise comparison of the data between successive biological states (for example, normal versus tumor), we clustered the expression data on the basis of behavior in multiple biological states, representative of the entire process of prostate cancer progression. By examining the data in the context of different proliferative conditions, before and after hormonal manipulation in the xenograft model, we could identify groups of genes associated with proliferation, metabolism, hormone responsiveness and malignant transformation, as well as a small number of genes directly associated with androgen-independent growth. Our results imply that the evolution to androgen independence is due, in part, to reactivation of the androgen-response pathway in the absence of androgens, but that this reactivation is probably incomplete.

Amler, Lukas

\section{Dysregulated expression of androgen- responsive and nonresponsive genes in the androgen-independent prostate cancer xenograft model, CWR22-R}

Lukas Amler $^{1}$, David Agus ${ }^{2}$, Carrie LeDuc ${ }^{1}$, Lisa Sapinoso ${ }^{1}$, William Fox ${ }^{2}$, Suzanne Kern ${ }^{1}$, Dori Lee ${ }^{1}$, Vivian Wang ${ }^{1}$,

Mauri Leysens ${ }^{1}$, Brian Higgins ${ }^{2}$, Jason Martin ${ }^{1}$,

William Herald ${ }^{3}$, Nicholas Dracopoli ${ }^{1}$, Carlos Cordon-Cardo ${ }^{3}$, Howard Scher ${ }^{2} \&$ Garret Hampton ${ }^{1}$

${ }^{1}$ GENOS Biosciences, Inc., La Jolla, California, USA

${ }^{2}$ Department of Medicine, Memorial Sloan-Kettering Cancer Center, New York, New York, USA

${ }^{3}$ Department of Pathology, Memorial Sloan-Kettering Cancer Center, New York, New York, USA

Treatment of metastatic prostate cancer with androgen ablation often elicits dramatic tumor regressions, but the response is rarely complete, making clinical recurrence inevitable with time. To gain insight into therapy-related progression, we used microarray analysis to monitor changes in gene expression that occurred following androgen starvation of an androgen-dependent prostate tumor xenograft, CWR22, and the emergence of an androgen-independent tumor, CWR22-R. Androgen deprivation arrested the growth of CWR22 cells, as evidenced by decreased expression of genes encoding cell cycle components and basal cell metabolism, respiration and transcription, as well as the induced expression of putative negative regulatory genes that may act to sustain cells in a nonproliferative state. Evolution of androgen-independent growth and proliferation, represented by CWR22-R, was associated with a re-entry into the active cell cycle and the upregulation of several genes that were expressed at low levels or absent in the androgen-dependent tumor. Restoration of androgen to mice bearing androgenindependent CWR22-R tumors induced, augmented or repressed the expression of several androgen-responsive genes. Two of the genes whose expression was increased by androgens (the calcium-binding protein, S100P, and the FK-506binding protein, FKBP51) were also expressed in CWR22-R. The dysregulated behavior of these genes is analogous to that of other androgen-dependent genes (for example, prostate-specific antigen and human kallikrein 2), which are commonly re-expressed in androgen-independent disease in the absence of androgens. These results imply that evolution to androgen independence is due in part to reactivation of the androgen response pathway in the absence of androgens, but that this reactivation is probably incomplete.

Aprelikova, Olga

[10]

\section{Differential gene expression in cells with targeted deletion of BRCA1 tumor suppressor}

\author{
Olga Aprelikova ${ }^{1}$, Amy Pace ${ }^{2}$, Beverly Koller $^{2} \&$ Edison Liu ${ }^{1}$ \\ ${ }^{1}$ Section of Molecular Signaling and Oncogenesis, Division of Clinical Sciences, \\ National Cancer Institute, Bethesda, Maryland 20892, USA \\ ${ }^{2}$ Lineberger Comprehensive Cancer Center, University of North Carolina at \\ Chapel Hill, Chapel Hill, North Carolina 27599, USA
}

$B R C A 1$ plays a role in DNA repair and transcription, although the importance of the transcriptional control to the clinical phenotype is unclear. To identify transcriptional targets of $B R C A 1$, we assessed differential gene expression in mouse embryonic stem cells with deletion of both BRCA1 alleles using high-density microarrays. Several groups of genes were downregulated in the knockout cell line, including some stress response genes, cytoskeleton genes and genes involved in protein synthesis and degradation. Of particular interest was the finding that the 14-3-3 $\sigma$ checkpoint control gene was significantly downregulated in $B R C A 1^{-1-}$ cells when compared with wild-type embryonic stem cells or $B R C A 1^{-1-}$ embryonic stem cells reconstituted with a $B R C A 1$ transgene. Irradiation of $B R C A 1^{-1-}$ cells showed much lower levels of 14-3-3 $\sigma$ induction. Moreover, when these cells were treated with ionizing radiation their cell cycle profile showed an inability to sustain the G2/M growth arrest typical of cells deprived of 14-3-3 $\sigma$. We found that $B R C A 1$ synergistically activates p53-dependent transcription of the 14-3-3 $\sigma$ gene and that this activation is dependent on an intact $\mathrm{p} 53, B R C A 1$ and $\mathrm{p} 53$ response elements. These data indicate that BRCA1 is an important transcriptional cofactor in p53-mediated responses to DNA damage and may explain some of the clinical and laboratory presentations of cancers in $B R C A 1$ carriers. 